\title{
NONLOCALIZED MODULATION OF PERIODIC REACTION DIFFUSION WAVES: NONLINEAR STABILITY
}

\author{
MATHEW A. JOHNSON, PASCAL NOBLE, L.MIGUEL RODRIGUES, AND KEVIN ZUMBRUN
}

\begin{abstract}
By a refinement of the technique used by Johnson and Zumbrun to show stability under localized perturbations, we show that spectral stability implies nonlinear modulational stability of periodic traveling-wave solutions of reaction diffusion systems under small perturbations consisting of a nonlocalized modulation plus a localized perturbation. The main new ingredient is a detailed analysis of linear behavior under modulational data $\bar{u}^{\prime}(x) h_{0}(x)$, where $\bar{u}$ is the background profile and $h_{0}$ is the initial modulation.
\end{abstract}

\section{INTRODUCTION}

Stability and behavior of modulated periodic wave trains have received considerable recent attention, both in the reaction diffusion and conservation law contexts; see, for example [S1, S2, DSSS, JZ1, JZ2, JZN, BJNRZ1, BJNRZ2, NR1, NR2] and references therein. The initial mathematical challenge of this problem is that the linearized equations, being periodic-coefficient, have purely essential spectrum when considered as problems on the whole line, so that there is no spectral gap between neutral and other modes, making difficult either the treatment of linearized behavior or the passage from linear to nonlinear estimates.

This issue was overcome in the reaction diffusion context in the late 1990s by Schneider [S1, S2], resolving an at the time 30-year open problem. Using a method of "diffusive stability," Schneider combined diffusive-type linear estimates with renormalization techniques to show that, assuming "diffusive" spectral stability in the sense to be specified later of the background periodic wave, long-time behavior under localized perturbations is essentially described by a scalar heat equation in the phase variable, with the amplitude decaying more rapidly.

More recently, Oh-Zumbrun and Johnson-Zumbrun [OZ1, JZ2] in 2010 using rather different tools coming from viscous shock theory have resolved the corresponding problem in the conservation law case, showing that, again assuming diffusive spectral stability of the background periodic wave, behavior under localized perturbations is described, roughly, by a system of viscous conservation laws in the derivative of the phase and other modulation parameters, so that the phase decays at slower, errorfunction rate than in the reaction diffusion case. This description has since been sharpened in [JNRZ2], showing that behavior consists of a modulation governed by the associated formal second-order Whitham averaged system plus a faster-decaying term. Further developments include the treatment of nonlinear stability of roll waves of the St. Venant equations [JZN, BJNRZ1] and the resolution in [BJNRZ2] of the 35-year open problem of proving nonlinear stability of spectrally stable periodic Kuramoto-Sivashinsky waves.

Applied to the reaction diffusion context [JZ1], the approach of [JZ2] recovers and slightly extends the results of Schneider, yielding the same heat kernel rate of decay with respect to localized perturbations while removing the assumption of $[\mathrm{S} 1, \mathrm{~S} 2]$ that nearby periodic waves have constant

Date: October 30, 2018.

Research of M.J. was partially supported by an NSF Postdoctoral Fellowship under NSF grant DMS-0902192.

Research of P.N. was partially supported by the French ANR Project no. ANR-09-JCJC-0103-01.

Stay of M.R. in Bloomington was supported by French ANR Project no. ANR-09-JCJC-0103-01.

Research of K.Z. was partially supported under NSF grant no. DMS-0300487. 
speed. However, one might hope to do better, based on the successful analysis in [JZ2], etc., in the conservation law case, of perturbations decaying at slower, errorfunction rate.

As described clearly in [DSSS], formal (WKB) asymptotics suggest the same thought, yielding the description to lowest order of a scalar viscous conservation law

$$
k_{t}-\omega(k)_{x}=\left(d(k) k_{x}\right)_{x}
$$

for the wave number $k=\psi_{x}$, where frequency $\omega=\psi_{t}$ and $\psi$ is the phase. Here, $\omega(k)=-c(k) k$ and wave speed $c(k)$ are determined through the nonlinear dispersion relation obtained from the manifold of periodic traveling-wave solutions $\bar{u}^{k}(k(x-c t))$, and $d(k)$ through higher-order asymptotics. See [Se, OZ2, NR1, NR2] for corresponding developments in the conservation law case. From (1.1) and the definition $k=\psi_{x}$, one might hope to treat initial modulations $\left.\psi\right|_{t=0}=h_{0}$ for which, not the phase $h_{0}$, but its derivative $\partial_{x} h_{0}$ is localized $\left(L^{1}\right)$, obtaining the same heat kernel rate of decay for $k$ as found in the conservation law case [JZ2].

In this note, we show that this is indeed the case, proving by an adaptation of the methods of [JZ1, JZ2] that diffusively spectrally stable periodic reaction diffusion waves are nonlinearly stable under initial perturbations consisting of a localized perturbation plus a nonlocalized modulation $h_{0}$, $\partial_{x} h_{0} \in L^{1}$, in phase, with heat kernel rate of decay in the wave number and errorfunction decay in the phase. In a companion paper [JNRZ1], we build on these basic estimates to establish, further, time-asymptotic behavior, validating description (1.1) in the long-time limit. Recall that (1.1) has been validated in [DSSS] in the related, asymptotically-large-bounded-time small-wavenumber limit and for data in uniformly-local Sobolev spaces ${ }^{1}$, in the somewhat different sense of showing that there exists a $\delta$-family of solutions of the full system $\delta^{M}$-close to a formal expansion ${ }^{2}$ in $\delta$ on intervals $\left[0, T / \delta^{2}\right]$, where $M$ and $T$ are arbitrarily large and $\delta$ is the characteristic size of the wavenumber of the modulation: that is, they build "prepared" data for bounded intervals rather than work with general ones globally in time.

Consider a periodic traveling-wave solution of reaction diffusion equation $u_{t}=u_{x x}+f(u)$, or, equivalently, a standing-wave solution $u(x, t)=\bar{u}(x)$ of

$$
k_{\star} u_{t}=k_{\star}^{2} u_{x x}+f(u)+k_{\star} c u_{x},
$$

where $c$ is the speed of the original traveling wave, and wave number $k_{*}$ is chosen so that

$$
\bar{u}(x+1)=\bar{u}(x) .
$$

For simplicity of notation, we will follow this convention throughout the paper; that is, all periodic functions are assumed to be periodic of period one.

We make the following standard genericity assumptions:

(H1) $f \in C^{K}(\mathbb{R}),(K \geq 3)$.

(H2) Up to translation, the set of 1-periodic solutions of (1.2) (with $k$ replacing $k_{\star}$ ) in the vicinity of $\bar{u}, k=k_{\star}$, forms a smooth 1-dimensional manifold $\{\bar{u}(k, \cdot)\}=\left\{\bar{u}^{k}(\cdot)\right\}, c=c(k)$.

Linearizing (1.2) about $\bar{u}$ yields the periodic coefficient equation

$$
k_{\star} v_{t}=k_{\star} L v:=\left(k_{\star}^{2} \partial_{x}^{2}+k_{\star} c \partial_{x}+b\right) v, \quad b(x):=d f(\bar{u}(x)) .
$$

Introducting the family of Floquet operators

$$
k_{\star} L_{\xi}:=e^{-i \xi x} k_{\star} L e^{i \xi x}=k_{\star}^{2}\left(\partial_{x}+i \xi\right)^{2}+k_{\star} c\left(\partial_{x}+i \xi\right)+b,
$$

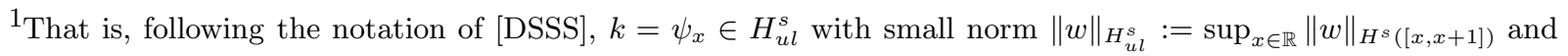
$s \geq M+2 \geq 4$.

${ }^{2}$ But this expansion is only $\delta^{2}$-close to the approximate solution involving only the solution of the second order Whitham equation.
} 
operating on periodic functions on $[0,1]$, determined by the defining relation

$$
L\left(e^{i \xi x} f\right)=e^{i \xi x}\left(L_{\xi} f\right) \text { for } f \text { periodic, }
$$

we define following $[\mathrm{S} 1, \mathrm{~S} 2]$ the standard diffusive spectral stability conditions:

(D1) $\lambda=0$ is a simple eigenvalue of $L_{0}$. (Note that $\xi=0$ corresponds to co-periodic perturbations, hence $L_{0}$ has always at least the translational, zero-eigenfunction $\bar{u}^{\prime}$.)

(D2) $\sigma\left(L_{\xi}\right) \subset\left\{\left.\lambda|\Re \lambda \leq-\theta| \xi\right|^{2}\right\}$ for some constant $\theta>0$.

Then, we have the following Main Theorem, extending the results of [JZ1] to nonlocalized perturbations.

Theorem 1.1. Let $K \geq 3$. Assuming (H1)-(H2) and (D1)-(D2), let

$$
E_{0}:=\left\|\tilde{u}_{0}\left(\cdot-h_{0}(\cdot)\right)-\bar{u}(\cdot)\right\|_{L^{1}(\mathbb{R}) \cap H^{K}(\mathbb{R})}+\left\|\partial_{x} h_{0}\right\|_{L^{1}(\mathbb{R}) \cap H^{K}(\mathbb{R})}
$$

be sufficiently small, for some choice of modulation $h_{0}$. Then, there exists a global solution $\tilde{u}(x, t)$ of (1.2) with initial data $\tilde{u}_{0}$ and a phase function $\psi(x, t)$ such that, for $t>0$ and $2 \leq p \leq \infty$,

$$
\begin{aligned}
\|\tilde{u}(\cdot-\psi(\cdot, t), t)-\bar{u}(\cdot)\|_{L^{p}(\mathbb{R})} & \lesssim E_{0}(1+t)^{-\frac{1}{2}(1-1 / p)} \\
\left\|\nabla_{x, t} \psi(\cdot, t)\right\|_{L^{p}(\mathbb{R})} & \lesssim E_{0}(1+t)^{-\frac{1}{2}(1-1 / p)},
\end{aligned}
$$

and

$$
\|\tilde{u}(\cdot, t)-\bar{u}(\cdot)\|_{L^{\infty}(\mathbb{R})}, \quad\|\psi(\cdot, t)\|_{L^{\infty}(\mathbb{R})} \lesssim E_{0} .
$$

In particular, $\bar{u}$ is nonlinearly (boundedly) stable in $L^{\infty}(\mathbb{R})$ with respect to initial perturbations $v_{0}=\tilde{u}_{0}-\bar{u}$ for which $\left\|v_{0}\right\|_{E}:=\inf _{\partial_{x} h_{0} \in L^{1}(\mathbb{R}) \cap H^{K}(\mathbb{R})} E_{0}$ is sufficiently small.

Remark 1.2. It may seem more natural, and indeed is so, to introduce $h_{0}$ and $\psi$ via

$$
v(x, 0)=\tilde{u}_{0}(x)-\bar{u}\left(x+h_{0}(x)\right), \quad v(x, t)=\tilde{u}(x, t)-\bar{u}(x+\psi(x, t)) .
$$

However, in doing so one introduces in the equation for $v$ terms involving only $\psi$ and thus not decaying in time; see Lemma 5.1 below. For this reason we work instead with

$$
v(x, t)=\tilde{u}(x-\psi(x, t), t)-\bar{u}(x),
$$

that is,

where $Y$ is such that

$$
\tilde{u}(x, t)=\bar{u}(Y(x, t))+v(Y(x, t), t)
$$

$$
Y(x, t)-\psi(Y(x, t), t)=x, \quad Y(y-\psi(y, t), t)=y .
$$

Notice that we insure the existence of such a map $Y$ by keeping, for any $t,\|\psi(\cdot, t)\|_{L^{\infty}(\mathbb{R})}$ bounded and $\left\|\psi_{x}(\cdot, t)\right\|_{L^{\infty}(\mathbb{R})}$ small. It should be stressed, however, that ${ }^{3}$

$$
Y(x, t)=x+\psi(x, t)+O\left(\|\psi(\cdot, t)\|_{L^{\infty}}\left\|\psi_{x}(\cdot, t)\right\|_{L^{\infty}}\right)
$$

so that we are not so far from the natural (but inappropriate) approach. Furthermore, notice also that introducing the map $Y$ above enables one to go back to the original unknown $\tilde{u}(x, t)$ when desired.

At a philosophical level, the main new observation here beyond what was shown in [JZ1] is that linear perturbations that are "asymptotically modulational" ${ }^{4}$ behave as regards the linear solution operator $S(t)$ as if they were asymptotically constant; in particular, $\partial_{x} S(t)\left(\bar{u}^{\prime} h_{0}\right) \sim S(t)\left(\bar{u}^{\prime} \partial_{x} h_{0}\right)$, where $\bar{u}$ is the background traveling wave (see Section 4). The analysis is based, at a technical level,

\footnotetext{
${ }^{3}$ This follows from $Y(X(y, t), t)-[X(y, t)+\psi(X(y, t), t)]=\psi(y, t)-\psi(y-\psi(y, t), t)$.

${ }^{4}$ In the sense that they converge at plus and minus spatial infinity to scalar multiples $h_{0} \bar{u}^{\prime}$ of the derivative $\bar{u}^{\prime}$ of the background traveling wave, corresponding at linear level to shifts $\bar{u}\left(x+h_{0}(x)\right)$.
} 
on the decomposition of Floquet modes of $\bar{u}^{\prime} h_{0}$ into the sum of Fourier modes of $h_{0}$ times the periodic function $\bar{u}^{\prime}(x)$, and the separate analysis/recombination of each frequency range $[-\pi+2 j \pi, \pi+2 j \pi]$. We are unable to see such detail from the Green function description of [JZ1, JZ2, JZN], nor from weighted energy estimates as in [S1, S2]. Indeed, this seems to be an instance where frequency domain techniques detect cancellation not readily apparent by spatial-domain techniques.

In the process, we obtain an alternative proof of the basic estimates in [JZ1], carried out entirely within the Bloch transform formulation without passing to a Green function description as in [JZ1]. This elucidates somewhat the relation between the Bloch tranform based estimates of [S1, S2] and the Green function based estimates of [JZ1, JZ2], in particular the role of the Green function (integral kernel) in obtaining the estimates of [JZ1]; see Remark 3.2.

Our analysis suggests that one might by an elaboration of the approach used here treat still more general perturbations converging asymptotically to any fixed periodic perturbations at $\pm \infty$, not necessarily modulations, which would then time-exponentially relax to modulations following the dynamics of the corresponding periodic problem; see Remark 4.2.2. This would be an interesting direction for further investigation.

We mention, finally, that the techniques introduced here are not limited to the reaction-diffusion case, but apply equally to the conservative case treated in [JZ2], yielding a comparable result of stability with respect to nonlocalized modulations [JNRZ3]. Indeed, this clarifies somewhat the relation between the reaction diffusion and conservation law case, revealing a continuum of models with common behavior lying between these two extremes. These issues will be reported on elsewhere.

Remark 1.3. Without change, we may treat reaction-diffusion systems with a diagonal diffusion as considered in [DSSS]. More generally, all proofs go through whenever the linearized operator generates a $C^{0}$ semigroup; in particular, our results apply to the (sectorial) Swift-Hohenberg equations treated for localized perturbations in [S2]..$^{5}$ It would be interesting to extend to the multi-dimensional case as in [U].

Note: We have been informed by Bjorn Sandstede that similar results have been obtained by different means by him and collaborators [SSSU] using a nonlinear decomposition of phase and amplitude variables as in [DSSS].

\section{Preliminaries}

Recall the Bloch solution formula for periodic-coefficient operators,

$$
(S(t) g)(x):=\left(e^{t L} g\right)(x)=\int_{-\pi}^{\pi} e^{i \xi x}\left(e^{t L_{\xi}} \check{g}(\xi, \cdot)\right)(x) d \xi,{ }^{6}
$$

where $L_{\xi}$ is as in (1.5),

$$
\check{g}(\xi, x):=\sum_{j \in \mathbb{Z}} \hat{g}(\xi+2 j \pi) e^{i 2 \pi j x},
$$

periodic, denotes the Bloch transform of $g, \hat{g}(\xi):=\frac{1}{2 \pi} \int_{\mathbb{R}} e^{-i \xi x} g(x) d x$ the Fourier transform, and

$$
g(x)=\int_{-\pi}^{\pi} e^{i \xi x} \check{g}(\xi, x) d \xi
$$

the inverse Bloch transform, or Bloch representation of $g \in L^{2}$.

\footnotetext{
${ }^{5}$ See [BJNRZ1, BJNRZ2, BJNRZ3, JZN] for related analyses in such general settings.

${ }^{6}$ In other words, $\left(e^{t \check{L}} g\right)(\xi, x)=\left(e^{t L_{\xi}} \check{g}(\xi, \cdot)\right)(x)$, a consequence of (1.6).
} 
Note that, in view of the inverse Bloch transform formula (2.3), the generalized Hausdorff-Young inequality $\|u\|_{L^{p}(\mathbb{R})} \leq\|\check{u}\|_{L^{q}\left(\xi, L^{p}([0,1])\right)}$ for $q \leq 2 \leq p$ and $\frac{1}{p}+\frac{1}{q}=1$ [JZ1], yields for any 1-periodic functions $f(\xi, \cdot), \xi \in[-\pi, \pi]$,

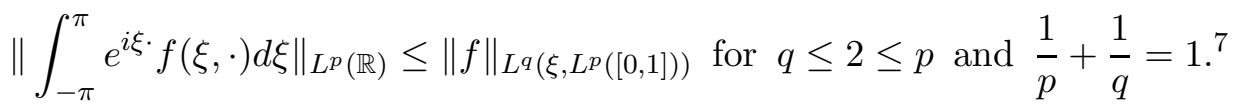

This convenient formulation is the one by which we will obtain all of our linear estimates.

Note by (D1) that there exists a simple zero eigenfunction $\bar{u}^{\prime}$ of $L_{0}$, which by standard perturbation results $[\mathrm{K}]$ thus bifurcates to an eigenfunction $\phi(\xi, \cdot)$, with associated left eigenfunction $\tilde{\phi}(\xi, \cdot)$ and eigenvalue

$$
\lambda(\xi)=a i \xi-d \xi^{2}+O\left(|\xi|^{3}\right),
$$

where $a$ and $d$ are real and $d>0$ by assumption (D2) and complex symmetry, $\lambda(\xi)=\bar{\lambda}(-\xi)$, each of $\phi, \tilde{\phi}, \lambda$ analytic in $\xi$ and defined for $|\xi|$ sufficiently small.

\section{BASIC LINEAR ESTIMATES}

Loosely following [JZ1] decompose the solution operator as

$$
S(t)=S^{p}(t)+\tilde{S}(t), \quad S^{p}(t)=\bar{u}^{\prime} s^{p}(t),
$$

with

$$
\left(s^{p}(t) g\right)(x)=\int_{-\pi}^{\pi} e^{i \xi x} \alpha(\xi) e^{\lambda(\xi) t}\langle\tilde{\phi}(\xi, \cdot), \check{g}(\xi, \cdot)\rangle_{L^{2}([0,1])} d \xi
$$

and

$$
\begin{aligned}
(\tilde{S}(t) g)(x) & :=\int_{-\pi}^{\pi} e^{i \xi x}(1-\alpha(\xi))\left(e^{L_{\xi} t} \check{g}(\xi)\right)(x) d \xi+\int_{-\pi}^{\pi} e^{i \xi x} \alpha(\xi)\left(e^{L_{\xi} t} \tilde{\Pi}(\xi) \check{g}(\xi)\right)(x) d \xi \\
& +\int_{-\pi}^{\pi} e^{i \xi x} \alpha(\xi) e^{\lambda(\xi) t}(\phi(\xi, x)-\phi(0, x))\langle\tilde{\phi}(\xi), \check{g}(\xi)\rangle_{L^{2}([0,1])} d \xi
\end{aligned}
$$

where $\alpha$ is a smooth cutoff function supported on $\xi$ sufficiently small, ${ }^{8}$

$$
\Pi^{p}(\xi):=\phi(\xi)\langle\tilde{\phi}(\xi), \cdot\rangle_{L^{2}([0,1])}
$$

defined for sufficiently small $\xi$ denotes the eigenprojection onto the eigenspace Range $\{\phi(\xi)\}$ bifurcating from Range $\left\{\bar{u}^{\prime}(x)\right\}$ at $\xi=0, \tilde{\phi}$ the associated left eigenfunction, and $\tilde{\Pi}:=\mathrm{Id}-\Pi^{p}$.

We begin by reproving the following estimates established (in a slightly different form) in [JZ1], describing linear behavior under a localized perturbation $g \in L^{1}(\mathbb{R}) \cap L^{2}(\mathbb{R})$.

Proposition 3.1 ([JZ1]). Under assumptions (H1)-(H2) and (D1)-(D2), for all $t>0,2 \leq p \leq \infty$,

$$
\left\|\partial_{x}^{l} \partial_{t}^{m} s^{p}(t) \partial_{x}^{n} g\right\|_{L^{p}(\mathbb{R})} \lesssim \min \left\{\begin{array}{l}
(1+t)^{-\frac{1}{2}(1-1 / p)-\frac{l+m}{2}}\|g\|_{L^{1}(\mathbb{R})}, \\
(1+t)^{-\frac{1}{2}(1 / 2-1 / p)-\frac{l+m}{2}}\|g\|_{L^{2}(\mathbb{R})}
\end{array}\right.
$$

for $0 \leq n \leq K+1$, and for some $\eta>0$ and $0 \leq l+2 m, n \leq K+1$,

$$
\left\|\partial_{x}^{l} \partial_{t}^{m} \tilde{S}(t) \partial_{x}^{n} g\right\|_{L^{p}(\mathbb{R})} \lesssim \min \left\{\begin{array}{l}
(1+t)^{-\frac{1}{2}(1-1 / p)-\frac{1}{2}}\|g\|_{L^{1}(\mathbb{R}) \cap H^{l+2 m+1}(\mathbb{R})}, \\
e^{-\eta t}\left\|\partial_{x}^{n} g\right\|_{H^{l+2 m+1}(\mathbb{R})}+(1+t)^{-\frac{1}{2}(1 / 2-1 / p)-\frac{1}{2}}\|g\|_{L^{2}(\mathbb{R})},
\end{array}\right.
$$

Estimates (3.5)-(3.6) were established in [JZ1] by first passing to a Green function formulation. Here, we give an alternative proof within the Bloch formulation that will be useful for what follows.

\footnotetext{
${ }^{7}$ Here, and elsewhere, we are adopting the notation $\|f\|_{L^{q}\left(\xi, L^{p}([0,1])\right)}:=\left(\int_{-\pi}^{\pi}\|f(\xi, \cdot)\|_{L^{p}([0,1])}^{q} d \xi\right)^{1 / q}$.

${ }^{8}$ That is, $\alpha=0$ for $|\xi| \geq 2 \xi_{0}$ and $\alpha=1$ for $|\xi| \leq \xi_{0}, \xi_{0}$ sufficiently small.
} 
Proof. (i) (Proof of (3.5)(1)). First, expand

$$
\begin{aligned}
\left(s^{p}(t) g\right)(x) & =\int_{-\pi}^{\pi} \alpha(\xi) e^{\lambda(\xi) t} e^{i \xi x}\langle\tilde{\phi}, \check{g}\rangle_{L^{2}([0,1])}(\xi) d \xi \\
& =\sum_{j \in \mathbb{Z}} \int_{-\pi}^{\pi} \alpha(\xi) e^{\lambda(\xi) t} e^{i \xi x}\left\langle\tilde{\phi}(\xi, y), e^{i 2 \pi j y}\right\rangle_{L^{2}([0,1])} \hat{g}(\xi+2 j \pi) d \xi \\
& =\sum_{j \in \mathbb{Z}} \int_{-\pi}^{\pi} \alpha(\xi) e^{\lambda(\xi) t} e^{i \xi x} \hat{\tilde{\phi}}_{j}(\xi)^{*} \hat{g}(\xi+2 j \pi) d \xi
\end{aligned}
$$

where $\hat{\tilde{\phi}}_{j}(\xi)$ denotes the $j$ th Fourier coefficient in the Fourier expansion of periodic function $\tilde{\phi}(\xi, \cdot)$, and $z^{*}=\bar{z}$ denotes complex conjugate.

By Hausdorff-Young's inequality, $|\hat{g}| \leq\|g\|_{L^{1}(\mathbb{R})}$, and by (D2), $\left|e^{\lambda(\xi) t} \alpha^{1 / 2}(\xi)\right| \leq e^{-\eta \xi^{2} t}, \eta>0$, while by by Cauchy-Schwarz' inequality,

$$
\alpha^{1 / 2}(\xi) \sum_{j}\left|\hat{\tilde{\phi}}_{j}(\xi)\right| \leq \alpha^{1 / 2}(\xi) \sqrt{\sum_{j}\left(1+|j|^{2}\right)\left|\hat{\tilde{\phi}}_{j}(\xi)\right|^{2} \sum_{j}\left(1+|j|^{-2}\right)} \leq C \alpha^{1 / 2}(\xi)\|\tilde{\phi}(\xi)\|_{H^{1}(\mathbb{R} ; d x)} .
$$

Combining these facts, and applying (2.4), we obtain for $1 / q+1 / p=1$

$$
\left\|s^{p}(t) g\right\|_{L^{p}(\mathbb{R} ; d x)} \lesssim\left\|e^{-\eta \xi^{2} t}\right\|_{L^{q}([-\pi, \pi], d \xi)} \sup _{|\xi| \leq \varepsilon}\|\tilde{\phi}\|_{H^{1}([0,1] ; d x)}\|g\|_{L^{1}(\mathbb{R})} \lesssim(1+t)^{-\frac{1}{2}(1-1 / p)}\|g\|_{L^{1}(\mathbb{R})},
$$

yielding the result for $l=m=n=0$. Estimates for general $l, m, n \geq 0$ go similarly, passing $\partial_{x}^{n}$ derivatives onto $\tilde{\phi}$ in the inner product using integration by parts and noting that $\partial_{x}^{l}$ and $\partial_{t}^{m}$ derivatives bring down harmless bounded factors $(i \xi)^{l}$ and $\lambda(\xi)^{m}$.

(ii) (Proof of (3.5)(2)). The second estimate on $s^{p}$ follows similarly, but substituting for the estimate of term $\int_{-\pi}^{\pi} e^{i \xi x} \alpha(\xi) e^{\lambda(\xi) t}\langle\tilde{\phi}(\xi), \check{g}(\xi)\rangle_{L^{2}([0,1])} d \xi$ the slightly simpler estimate

$$
\begin{aligned}
\left\|\int_{-\pi}^{\pi} e^{i \xi x} \alpha(\xi) e^{\lambda(\xi) t}\langle\tilde{\phi}, \check{g}\rangle_{L^{2}([0,1])} d \xi\right\|_{L^{p}(\mathbb{R} ; d x)} & \lesssim\left\|\alpha(\xi) e^{-\eta \xi^{2} t}\left|\langle\tilde{\phi}(\xi), \check{g}(\xi)\rangle_{L^{2}([0,1])}\right|\right\|_{L^{q}\left(\xi, L^{p}([0,1])\right)} \\
& \lesssim\left\|e^{-\eta \xi^{2} t}\right\| \check{g}(\xi)\left\|_{L^{2}([0,1] ; d x)}\right\|_{L^{q}([-\pi, \pi] ; d \xi)} \\
& \leq\left\|e^{-\eta \xi^{2} t}\right\|_{L^{r q}([-\pi, \pi] ; d \xi)}\|g\|_{L^{2}(\mathbb{R} ; d x)} \\
& \lesssim(1+t)^{-\frac{1}{2}(1 / 2-1 / p)}\|g\|_{L^{2}(\mathbb{R})},
\end{aligned}
$$

where $1 / r+1 / s=1$ and $q s=2$, so that $r q=\frac{2}{2-q}$ is $\infty$ for $p=q=2$ and 2 for $q=1, p=\infty$. This verifies the result for $l=m=n=0$; estimates for general $l, m, n \geq 0$ go similarly.

(ii) (Proof of (3.6)(1)). By (D2) and Prüss' Theorem [Pr], we have

$$
\left|e^{L_{\xi} t}(1-\alpha(\xi))\right|_{H^{l+1}([0,1] ; d x) \rightarrow H^{l+1}([0,1] ; d x)}, \quad\left|\alpha(\xi) e^{L_{\xi} t} \tilde{\Pi}\right|_{H^{l+1}([0,1] ; d x) \rightarrow H^{l+1}([0,1] ; d x)} \lesssim e^{-\eta t}, \quad \eta>0,
$$

whence, by Sobelev embedding,

$$
\left|\partial_{x}^{l} e^{L_{\xi} t}(1-\alpha(\xi))\right|_{H^{l+1}([0,1] ; d x) \rightarrow L^{p}([0,1] ; d x)}, \quad\left|\partial_{x}^{j} \alpha(\xi) e^{L_{\xi} t} \tilde{\Pi}\right|_{H^{l+1}([0,1] ; d x) \rightarrow L^{p}([0,1] ; d x)} \lesssim e^{-\eta t}
$$

for $2 \leq p \leq \infty$; see [JZ1] for details. ${ }^{9}$ The $W^{l, p}(\mathbb{R})$ norms of the first two terms of $(3.3)$, by $(2.4)$ and Parseval's identity, $\|\check{g}\|_{L^{2}\left(\xi, H^{l+1}([0,1])\right)} \sim\|g\|_{H^{l+1}(\mathbb{R})},{ }^{10}$ are thus bounded by $C e^{-\eta t}\|g\|_{H^{l+1}(\mathbb{R})}$. The $W^{l, p}(\mathbb{R})$ norm of the third term may be bounded similarly as in the estimation of $s^{p}$ above,

\footnotetext{
${ }^{9}$ Here, we are using the fact (see for example the Evans function analysis of $[G]$ ) that $H^{l+1}(\mathbb{R})$ and $L^{2}(\mathbb{R})$ spectra coincide.

${ }^{10}$ More precisely,

$$
\|g\|_{H^{l+1}(\mathbb{R})}^{2}=\|\check{g}\|_{L^{2}\left(\xi, L^{2}([0,1])\right)}^{2}+\|\check{g}\|_{L^{2}\left(\xi, \dot{H}_{\xi}^{l+1}([0,1])\right)}^{2}:=\|\check{g}\|_{L^{2}\left(\xi, L^{2}([0,1])\right)}^{2}+\left\|\left(\partial_{x}+i \xi\right)^{l+1} \check{g}(\xi)\right\|_{L^{2}\left(\xi, L^{2}([0,1])\right)}^{2} .
$$
}


noting that the factor $(\phi(\xi)-\phi(0)) \sim|\xi|$ introduces an additional factor of $(1+t)^{-1 / 2}$ decay. This establishes the result for $m=n=0$; other cases go similarly, noting that $\partial_{t} e^{L_{\xi} t} \tilde{\Pi}=L_{\xi} e^{L^{\xi}} \tilde{\Pi}$, with $L_{\xi}$ a second-order operator, so that we may essentially trade one $t$-derivative for two $x$-derivatives.

(ii) (Proof of (3.6)(2)). The second estimate on $\tilde{S}$ follows similarly, but substituting for the estimate of term $\int_{-\pi}^{\pi} e^{i \xi x} \alpha(\xi) e^{\lambda(\xi) t}(\phi(\xi, x)-\phi(0, x))\langle\tilde{\phi}(\xi), \check{g}(\xi)\rangle_{L^{2}([0,1])} d \xi$ the estimate

$$
\begin{aligned}
\left\|\int_{-\pi}^{\pi} e^{i \xi x} \alpha(\xi) e^{\lambda(\xi) t}(\phi(\xi, x)-\phi(0, x))\langle\tilde{\phi}(\xi), \check{g}(\xi)\rangle d \xi\right\|_{L^{p}(x)} & \lesssim\left\|\alpha ( \xi ) e ^ { - \eta \xi ^ { 2 } t } \left|\xi\|\langle\tilde{\phi}(\xi), \check{g}(\xi)\rangle \mid\|_{L^{q}\left(\xi, L^{p}(x)\right)}\right.\right. \\
& \lesssim\left\||\xi| e^{-\eta \xi^{2} t}\right\| \check{g}(\xi)\left\|_{L^{2}(x)}\right\|_{L^{q}(\xi)} \\
& \leq\left\||\xi| e^{-\eta \xi^{2} t}\right\|_{L^{r q}(\xi)}\|g\|_{L^{2}(x)} \\
& \lesssim(1+t)^{-\frac{1}{2}(1 / 2-1 / p)-\frac{1}{2}}\|g\|_{L^{2}}
\end{aligned}
$$

where $1 / r+1 / s=1$ and $q s=2$, so that $r q=\frac{2}{2-q}$ is $\infty$ for $p=q=2$ and 2 for $q=1, p=\infty$.

Remark 3.2. In [JZ1], by contrast, $s^{p}(t)$ is estimated through its integral kernel

$$
e(x, t ; y):=\left(s^{p}(t) \delta_{y}\right)(x)=\int_{-\pi}^{\pi} \alpha(\xi) e^{\lambda(\xi) t} e^{i \xi x} \tilde{\phi}(\xi, y) d \xi
$$

yielding the slightly sharper estimate $\frac{\left\|s^{p}(t) g\right\|_{L^{p}(x)}}{\|g\|_{L^{1}(x)}} \leq \sup _{y}\|e(\cdot, t ; y)\|_{L^{p}(x)} \leq\|\tilde{\phi}\|_{L^{\infty}(x, \xi)}(1+t)^{-1 / 2(1-1 / p)}$. For our purposes, this makes no difference, and, as we shall see in Section 4 , there can be an advantage in maintaining the separation into distinct frequencies afforded by the Bloch representation.

\section{Linear BEHAVior FOR MODUlational DATA}

Next, we consider behavior of (2.1) when applied to modulational data $g=h_{0} \bar{u}^{\prime}$, the linearized version of a nonlinear modulational perturbation $\bar{u}\left(x+h_{0}(x)\right)-\bar{u}(x) \sim h_{0}(x) \bar{u}^{\prime}(x)$. The following estimates, obtained by frequency-domain rather than spatial-domain (Green function) techniques as in [JZ1, JZ2, JZN], together with the associated modified nonlinear iteration scheme of Section 7 , below, represent the main new technical contributions of this paper.

Proposition 4.1. Under assumptions (H1)-(H2) and (D1)-(D2), for all $t>0,2 \leq p \leq \infty$,

$$
\left\|\partial_{x}^{l} \partial_{t}^{m} s^{p}(t)\left(h_{0} \bar{u}^{\prime}\right)\right\|_{L^{p}(\mathbb{R})} \lesssim(1+t)^{-\frac{1}{2}(1-1 / p)+\frac{1}{2}-\frac{l+m}{2}}\left\|\partial_{x} h_{0}\right\|_{L^{1}(\mathbb{R})},
$$

for $l+m \geq 1$ or else $l=m=0$ and $p=\infty$, and, for $0 \leq l+2 m \leq K+1$,

$$
\left\|\partial_{x}^{l} \partial_{t}^{m} \tilde{S}(t)\left(h_{0} \bar{u}^{\prime}\right)\right\|_{L^{p}(\mathbb{R})} \lesssim(1+t)^{-\frac{1}{2}(1-1 / p)}\left\|\partial_{x} h_{0}\right\|_{L^{1}(\mathbb{R}) \cap H^{l+2 m+1}(\mathbb{R})},
$$

and when $t \leq 1$

$$
\begin{aligned}
\left\|\partial_{x}^{l} \partial_{t}^{m}\left(S^{p}(t)-\mathrm{Id}\right)\left(h_{0} \bar{u}^{\prime}\right)\right\|_{L^{p}(\mathbb{R})} & \lesssim\left\|\partial_{x} h_{0}\right\|_{L^{1}(\mathbb{R}) \cap H^{l+2 m+1}(\mathbb{R})} \\
\left\|\partial_{x}^{l} \partial_{t}^{m}\left(s^{p}(t)\left(h_{0} \bar{u}^{\prime}\right)-h_{0}\right)\right\|_{L^{p}(\mathbb{R})} & \lesssim\left\|\partial_{x} h_{0}\right\|_{L^{1}(\mathbb{R}) \cap L^{2}(\mathbb{R})}
\end{aligned}
$$


Proof. (i) (Proof of (4.1)), $l+m \geq 1$. We treat the case $l=1, m=0$; other cases go similarly. First, re-express

$$
\begin{aligned}
\partial_{x}\left(s^{p}(t)\left(\bar{u}^{\prime} h_{0}\right)\right)(x) & =\int_{-\pi}^{\pi} i \xi \alpha(\xi) e^{\lambda(\xi) t} e^{i \xi x}\left\langle\tilde{\phi} \bar{u}^{\prime}, \check{h}_{0}\right\rangle_{L^{2}([0,1])}(\xi) d \xi \\
& =\sum_{j \in \mathbb{Z}} \int_{-\pi}^{\pi} i \xi \alpha(\xi) e^{\lambda(\xi) t} e^{i \xi x}\left\langle\tilde{\phi}(\xi, y) \bar{u}^{\prime}(y), e^{i 2 \pi j y}\right\rangle_{L^{2}([0,1])} \hat{h}_{0}(\xi+2 j \pi) d \xi \\
& =\sum_{j \in \mathbb{Z}} \int_{-\pi}^{\pi} i \xi \alpha(\xi) e^{\lambda(\xi) t} e^{i \xi x}{\widehat{\tilde{\phi}(\xi) \bar{u}^{\prime}}}_{j}^{*} \hat{h}_{0}(\xi+2 j \pi) d \xi \\
& =\sum_{j \in \mathbb{Z}} \int_{-\pi}^{\pi} \frac{\xi}{\xi+2 \pi j} \alpha(\xi) e^{\lambda(\xi) t} e^{i \xi x}{\widehat{\tilde{\phi}(\xi) \bar{u}^{\prime}}}_{j}^{*} \widehat{\partial_{x} h_{0}}(\xi+2 j \pi) d \xi,
\end{aligned}
$$

where $\widehat{\tilde{\phi}(\xi) \bar{u}^{\prime}}{ }_{j}$ denotes the $j$ th Fourier coefficient in the Fourier expansion of periodic function $\tilde{\phi}(\xi) \bar{u}^{\prime}$. By (2.4), we thus get $\left\|\partial_{x}\left(s^{p}(t)\left(\bar{u}^{\prime} h_{0}\right)\right)\right\|_{L^{p}(\mathbb{R})} \leq C(1+t)^{-\frac{1}{2}(1-1 / p)}\left\|\partial_{x} h_{0}\right\|_{L^{1}(\mathbb{R})} \sum_{j}\left|\frac{\widehat{\tilde{\phi}(\xi) \bar{u}^{\prime}}}{1+|j|}\right|$, yielding the result together with the Cauchy-Schwarz estimate

$$
\sum_{j}\left|\frac{\widehat{\tilde{\phi}(\xi) \bar{u}_{j}^{\prime}}}{1+|j|}\right| \leq \sqrt{\sum_{j}(1+|j|)^{-2} \sum_{j}\left|\widehat{\tilde{\phi}(\xi) \bar{u}_{j}^{\prime}}\right|^{2}} \lesssim\left\|\tilde{\phi}(\xi) \bar{u}^{\prime}\right\|_{L^{2}(\mathbb{R})} .
$$

(ii) (Proof of (4.1)), $l=m=0$. This follows by an explicit error function decomposition as in [JZ2], p. 18, putting a $\xi$ factor on $h_{0} \bar{u}^{\prime}$ and a $1 / \xi$ factor on $e^{\lambda(\xi) t}$ and proceeding as in the cited estimate. More precisely, for the low-frequency part of $\hat{h}_{0}$, express the principal part $\int_{-\pi}^{\pi} e^{i \xi x} \frac{e^{\left(a i \xi-d \xi^{2}\right) t}}{\xi} d \xi$ as

$$
\int_{-\infty}^{+\infty} e^{i \xi x} \frac{e^{\left(a i \xi-d \xi^{2}\right) t}}{i \xi} d \xi=\operatorname{errfn}\left((x-a t)^{2} / \sqrt{t}\right)
$$

minus $\sqrt{t} \int_{|\xi| \geq \pi} e^{i \xi x} \frac{e^{\left(a i \xi-d \xi^{2}\right) t}}{\xi t^{1 / 2}} d \xi$, estimating the $L^{\infty}(\mathbb{R})$ norm of convolutions with the former by the Triangle Inequality, and with the latter by Hausdorff-Young's inequality; for details, see [JZ2]. ${ }^{11}$

(iii) (Proof of (4.2)). Likewise, this follows by re-expressing

$$
\begin{aligned}
\tilde{S}(t)\left(h_{0} \bar{u}^{\prime}\right)(x)= & \int_{-\pi}^{\pi} e^{i \xi x}(1-\alpha(\xi))\left(e^{L_{\xi} t} \check{h}_{0}(\xi) \bar{u}^{\prime}\right)(x) d \xi+\int_{-\pi}^{\pi} e^{i \xi x} \alpha(\xi)\left(e^{L_{\xi} t} \tilde{\Pi}(\xi) \check{h}_{0}(\xi) \bar{u}^{\prime}\right)(x) d \xi \\
& +\int_{-\pi}^{\pi} e^{i \xi x} \alpha(\xi) e^{\lambda(\xi) t}(\phi(\xi, x)-\phi(0, x))\left\langle\tilde{\phi}(\xi), \check{h}_{0}(\xi) \bar{u}^{\prime}\right\rangle_{L^{2}([0,1])} d \xi \\
= & \sum_{j \in \mathbb{Z}} \int_{-\pi}^{\pi} e^{i \xi x}(1-\alpha(\xi)) \frac{\left(e^{L_{\xi} t}\left(\bar{u}^{\prime} e^{2 i \pi j}\right)\right)(x)}{i(\xi+2 \pi j)} \widehat{\partial_{x} h_{0}}(\xi+2 j \pi) d \xi \\
& +\sum_{j \in \mathbb{Z}} \int_{-\pi}^{\pi} e^{i \xi x} \alpha(\xi) \frac{\left(e^{L_{\xi} t} \tilde{\Pi}(\xi)\left(\bar{u}^{\prime} e^{2 i j \pi \cdot}\right)\right)(x)}{i(\xi+2 \pi j)} \widehat{\partial_{x} h_{0}}(\xi+2 j \pi) d \xi \\
& +\sum_{j \in \mathbb{Z}} \int_{-\pi}^{\pi} e^{i \xi x} \alpha(\xi) e^{\lambda(\xi) t} \frac{(\phi(\xi, x)-\phi(0, x)) \widetilde{\phi} \bar{u}_{j}^{\prime}(\xi)^{*}}{i(\xi+2 \pi j)} \widehat{\partial_{x} h_{0}}(\xi+2 j \pi) d \xi
\end{aligned}
$$

\footnotetext{
${ }^{11}$ This estimate is not needed for the nonlinear iteration, so we do not emphasize it here. We note that the resulting bounds (1.8) may be recovered alternatively by the modulation decomposition of [JNRZ1] without case $l=0$.
} 
then estimating as before, where we are using $(1-\alpha(\xi)) \lesssim \xi, \tilde{\Pi}(\xi) \bar{u}^{\prime}=O(\xi), \phi(\xi)-\phi(0)=O(\xi)$, respectively, to bound the key terms $\frac{(1-\alpha(\xi))}{i(\xi+2 \pi j)}, \frac{\tilde{\Pi}(\xi)\left(\bar{u}^{\prime} e^{2 i j \pi \cdot}\right)}{i(\xi+2 \pi j)}$, and $\frac{\phi(\xi)-\phi(0)}{i(\xi+2 \pi j)}$ for $j=0$, and are using the Cauchy-Schwarz inequality

$$
\sum_{j}\left|\frac{\widehat{\partial_{x} h_{0}}(\xi+2 j \pi)}{\xi+2 j \pi j}\right| \leq C \sqrt{\sum_{j} \frac{1}{(1+|j|)^{2}} \sum_{j^{\prime}}\left|\widehat{\partial_{x} h_{0}}\left(\xi+2 j^{\prime} \pi\right)\right|^{2}} \leq C\left\|\partial_{x} h_{0}\right\|_{L^{2}(\mathbb{R})}
$$

to bound the sum over $j \neq 0$. This establishes the case $l=m=0$; other cases go similarly.

(iv) (Proof of (4.3)). Expanding $S^{p}(t)-\mathrm{Id}=\left(S^{p}(t)-S^{p}(0)\right)-\tilde{S}(0)=t \partial_{t} S^{p}(s(t))-\tilde{S}(0)$ for some $0<s(t)<t$, we obtain the first inequality by combining (4.1) and (4.2). Likewise, the second ineqality follows by expanding $s^{p}(t)\left(h_{0} \bar{u}^{\prime}\right)-h_{0}=\left(s^{p}(t)-s^{p}(0)\right)\left(h_{0} \bar{u}^{\prime}\right)+\left(s^{p}(0)\left(h_{0} \bar{u}^{\prime}\right)-h_{0}\right)$ and applying (4.1) together with

$$
\begin{aligned}
& \left\|s^{p}(0)\left(h_{0} \bar{u}^{\prime}\right)-h_{0}\right\|_{L^{p}(\mathbb{R})} \\
& =\left\|\int_{-\pi}^{\pi} e^{i \xi x}(1-\alpha(\xi)) \sum_{j} \hat{h}_{0}(\xi+2 j \pi){\widehat{\widetilde{\phi}(\xi) \bar{u}^{\prime}}}_{j}^{*} d \xi+\int_{-\pi}^{\pi} e^{i \xi x} \sum_{j \neq 0} \hat{h}_{0}(\xi+2 j \pi)\left(\widehat{\tilde{\phi}(\xi)}^{*}{ }_{j}^{*}-1\right) d \xi\right\|_{L^{p}(\mathbb{R})} \\
& \leq C \sup _{\xi} \sum_{j} \frac{|\xi|\left|\tilde{\phi}(\xi) \bar{u}^{\prime}\right|}{|\xi+2 j \pi|}\left\|\partial_{x} h_{0}\right\|_{L^{1}(\mathbb{R})}+C \sup _{\xi} \sum_{j \neq 0} \frac{\left|\widehat{\partial_{x} h_{0}}(\xi+2 j \pi)\right|}{|\xi+2 j \pi|} \leq C\left\|\partial_{x} h_{0}\right\|_{L^{1}(\mathbb{R}) \cap L^{2}(\mathbb{R})}
\end{aligned}
$$

This establishes the case $l=m=0$; other cases go similarly.

Remark 4.2. 1. If we split $h_{0}$ into high-frequency and low-frequency parts, then the contribution of the high-frequency part decays faster by factor $(1+t)^{-1 / 2}$ in all estimates $(4.1)-(4.3)$.

2. In the estimate of $s^{p}$, it is easy to see that the same bounds hold if $\bar{u}^{\prime}$ is replaced by any periodic $f \in H_{\mathrm{per}}^{1}([0,1])$. However, in the estimate of $\tilde{S}$, replacing $\bar{u}^{\prime}$ by periodic $f \in H_{\mathrm{per}}^{1}([0,1])$ introduces an exponentially decaying error in $f-\bar{u}^{\prime}$, reflecting the dynamics of the problem on a periodic domain. To extend our results to the sum of a localized perturbaton and a perturbation asymptotic as $x \rightarrow \pm \infty$ to any two fixed periodic waves, not necessarily modulations, requires only the estimation of this latter error term as a time-exponentially function from $L^{\infty}(\mathbb{R}) \rightarrow L^{\infty}(\mathbb{R})$, a semigroup/Fourier multiplier problem of concrete technical nature. This would be an interesting direction for further investigation.

3. The estimate (4.3) is the key to handling the "initial layer problem" in our later nonlinear iteration, allowing us to essentially prescribe initial data for $\psi$ as is convenient for the analysis; see Remark 7.2 below for more information.

\section{Nonlinear Perturbation Equations}

Essentially following [JZ1], for $\tilde{u}(x, t)$ satisfying $k_{\star} \tilde{u}_{t}=k_{\star}^{2} \tilde{u}_{x x}+f(\tilde{u})+k_{\star} c \tilde{u}_{x}$ and $\psi(x, t)$ to be determined, set

$$
u(x, t)=\tilde{u}(x-\psi(x, t), t) \quad \text { and } \quad v(x, t)=u(x, t)-\bar{u}(x) .
$$

Lemma 5.1 ([JZ1]). The nonlinear residual $v$ defined in (5.1) satisfies

$$
k_{\star}\left(\partial_{t}-L\right)\left(v+\psi \bar{u}_{x}\right)=k_{\star} \mathcal{N}, \quad k_{\star} \mathcal{N}=\mathcal{Q}+\mathcal{R}_{x}+\left(k_{\star} \partial_{t}+k_{\star}^{2} \partial_{x}^{2}\right) \mathcal{S}+\mathcal{T},
$$

where

$$
\begin{gathered}
\mathcal{Q}:=f(v+\bar{u})-f(\bar{u})-d f(\bar{u}) v \\
\mathcal{R}:=-k_{\star} v \psi_{t}-k_{\star}^{2} v \psi_{x x}+k_{\star}^{2}\left(\bar{u}_{x}+v_{x}\right) \frac{\psi_{x}^{2}}{1-\psi_{x}}, \\
\mathcal{S}:=v \psi_{x} \\
9
\end{gathered}
$$


and

$$
\mathcal{T}:=-(f(v+\bar{u})-f(\bar{u})) \psi_{x} .
$$

Proof. (See also [JZ1]. ${ }^{12}$ ) From definition (5.1) and the fact that $\tilde{u}$ satisfies (1.2), we obtain

$$
k_{\star}\left(1-\psi_{x}\right) u_{t}+k_{\star}\left(-c+\psi_{t}\right) u_{x}=k_{\star}^{2}\left(\frac{1}{1-\psi_{x}} u_{x}\right)_{x}+\left(1-\psi_{x}\right) f(u)
$$

hence, subtracting the profile equation $-k_{*} c \bar{u}_{x}=k_{*}^{2} \bar{u}_{x x}+f(\bar{u})$ for $\bar{u}$,

$$
k_{\star} v_{t}-k_{\star} L v+k_{\star} \psi_{t} v_{x}-k_{\star} \psi_{x} v_{t}+k_{\star} \psi_{t} \bar{u}_{x}=\mathcal{Q}-\psi_{x} f(u)+k_{\star}^{2}\left(\frac{\psi_{x}}{1-\psi_{x}} u_{x}\right)_{x}
$$

or

$$
k_{\star}\left(\partial_{t}-L\right) v+k_{\star}\left(\psi \bar{u}_{x}\right)_{t}=\mathcal{Q}+\mathcal{R}_{x}+\left(k_{\star} \partial_{t}+k_{\star}^{2} \partial_{x}^{2}\right) \mathcal{S}-\psi_{x} f(u)+k_{\star}^{2}\left(\psi_{x} \bar{u}_{x}\right)_{x},
$$

and we finish the proof using $L \bar{u}_{x}=0$ and the profile equation for $\bar{u}$ to obtain

$$
k_{\star} L\left(\psi \bar{u}_{x}\right)=k_{\star} c \psi_{x} \bar{u}_{x}+k_{\star}^{2} \psi_{x} \bar{u}_{x x}+k_{\star}^{2}\left(\psi_{x} \bar{u}_{x}\right)_{x}=-\psi_{x} f(\bar{u})+k_{\star}^{2}\left(\psi_{x} \bar{u}_{x}\right)_{x} .
$$

\section{Nonlinear Damping estimate}

Proposition 6.1 ([JZ1]). Assuming $(H 1)-(H 2)$, let $v(\cdot, 0) \in H^{K}(\mathbb{R})$ (for $v$ as in (5.1)) and suppose that for some $T>0$, the $H^{K}(\mathbb{R})$ norm of $v(t)$ and $\psi_{t}(t)$ and the $H^{K+1}(\mathbb{R})$ norm of $\psi_{x}(t)$ remain bounded by a sufficiently small constant for all $0 \leq t \leq T$. Then there are positive constant $\theta$ and $C$, independent of $T$, such that, for all $0 \leq t \leq T$,

$$
\|v(t)\|_{H^{K}(\mathbb{R})}^{2} \leq C e^{-\theta t}\|v(0)\|_{H^{K}(\mathbb{R})}^{2}+C \int_{0}^{t} e^{-\theta(t-s)}\left(\|v(s)\|_{L^{2}(\mathbb{R})}^{2}+\left\|\left(\psi_{t}, \psi_{x}\right)(s)\right\|_{H^{K}(\mathbb{R})}^{2}\right) d s .
$$

Proof. Take for writing simplicity $k_{*}=1$. Rewriting (5.9) as

$$
\begin{aligned}
\left(1-\psi_{x}\right) v_{t}-v_{x x}-c v_{x}= & d f(\bar{u}) v+\mathcal{Q}-\left(\bar{u}_{x}+v_{x}\right) \psi_{t} \\
& +\left(\left(\bar{u}_{x}+v_{x}\right) \psi_{x}\right)_{x}+\left(\left(\bar{u}_{x}+v_{x}\right) \frac{\psi_{x}^{2}}{1-\psi_{x}}\right)_{x}-f(\bar{u}+v) \psi_{x},
\end{aligned}
$$

taking the $L^{2}$ inner product against $\sum_{j=0}^{K} \frac{(-1)^{j} \partial_{x}^{2 j} v}{1-\psi_{x}}$, integrating by parts, and rearranging, we obtain

$$
\frac{d}{d t}\|v\|_{H^{K}(\mathbb{R})}^{2}(t) \leq-\tilde{\theta}\left\|\partial_{x}^{K+1} v(t)\right\|_{L^{2}(\mathbb{R})}^{2}+C\left(\|v(t)\|_{H^{K}(\mathbb{R})}^{2}+\left\|\left(\psi_{t}, \psi_{x}\right)(t)\right\|_{H^{K}(\mathbb{R})}^{2}\right),
$$

for some $\tilde{\theta}>0$, so long as $\left\|\left(v, \psi_{t}, \psi_{x}, \psi_{x x}\right)(t)\right\|_{H^{K+1}(\mathbb{R})}$ remains sufficiently small. Sobolev interpolation $\|g\|_{H^{K}(\mathbb{R})}^{2} \leq \tilde{C}^{-1}\left\|\partial_{x}^{K+1} g\right\|_{L^{2}(\mathbb{R})}^{2}+\tilde{C}\|g\|_{L^{2}(\mathbb{R})}^{2}$ gives, then, for $\tilde{C}>0$ sufficiently large,

$$
\frac{d}{d t}\|v\|_{H^{K}(\mathbb{R})}^{2}(t) \leq-\theta\|v(t)\|_{H^{K}(\mathbb{R})}^{2}+C\left(\|v(t)\|_{L^{2}(\mathbb{R})}^{2}+\left\|\left(\psi_{t}, \psi_{x}\right)(t)\right\|_{H^{K}(\mathbb{R})}^{2}\right),
$$

from which (6.1) follows by Gronwall's inequality. See [JZ1] for further details.

\footnotetext{
${ }^{12}$ Note that we have here followed a different convention than in [JZ1], reversing the sign of $\psi$ in (5.1) in agreement with formal asymptotics of [Se, DSSS]. The change $\psi \rightarrow-\psi$ recovers the formulae of [JZ1].
} 


\section{NONLINEAR ITERATION SCHEME}

The key idea is, similarly as in the localized case treated in [JZ1], starting with

$$
\left(\partial_{t}-L\right)\left(v+\psi \bar{u}^{\prime}\right)=\mathcal{N},\left.\quad v\right|_{t=0}=d_{0},\left.\psi\right|_{t=0}=h_{0},
$$

where $d_{0}:=\tilde{u}_{0}\left(\cdot-h_{0}(\cdot)\right)-\bar{u} \in L^{1}(\mathbb{R}) \cap H^{K}(\mathbb{R}), \partial_{x} h_{0} \in L^{1}(\mathbb{R}) \cap H^{K}(\mathbb{R})$, to choose $\psi$ to cancel $s^{p}$ contributions, as

$$
\begin{aligned}
\psi(t) & =s^{p}(t)\left(h_{0} \bar{u}^{\prime}+d_{0}\right)+\int_{0}^{t} s^{p}(t-s) \mathcal{N}(s) d s \\
& -(1-\chi(t))\left(s^{p}(t)\left(d_{0}+h_{0} \bar{u}^{\prime}\right)-h_{0}+\int_{0}^{t} s^{p}(t-s) \mathcal{N}(s) d s\right),
\end{aligned}
$$

where $\chi(t)$ is a smooth cutoff that is zero for $t \leq 1 / 2$ and one for $t \geq 1$, leaving the system

$$
\begin{aligned}
v(t)= & \tilde{S}(t)\left(d_{0}+h_{0} \bar{u}^{\prime}\right)+\int_{0}^{t} \tilde{S}(t-s) \mathcal{N}(s) d s \\
& +(1-\chi(t))\left(S^{p}(t) d_{0}+\left(S^{p}(t)-\mathrm{Id}\right) h_{0} \bar{u}^{\prime}+\int_{0}^{t} S^{p}(t-s) \mathcal{N}(s) d s\right) .
\end{aligned}
$$

We may extract from (7.1)-(7.2) a closed system in $\left(v, \psi_{x}, \psi_{t}\right)$ (and some of their derivatives), and then recover $\psi$ through the slaved equation (7.1).

Remark 7.1. At first sight, we have accomplished nothing by introducing a $\psi$-dependent change of variable and choosing $\psi(0)=h_{0}$, since we have replaced the nonlocalized perturbation $\tilde{u}_{0}(x)-\bar{u}(x)$ used in the previous $h_{0}=0$ setup of [JZ1], by a different nonlocalized perturbation $d_{0}+h_{0} \bar{u}^{\prime}$. However, what we really did was replace the asymptotic states $\bar{u}\left(x+c_{ \pm}\right)-\bar{u}(x)$ by their linear approximates $c_{ \pm} \bar{u}^{\prime}(x)$, which removes the key difficulty of higher order remainders in the Taylor expansion of nonlinear modulations.

Remark 7.2. Notice that modulational data $\bar{u}^{\prime} h_{0}$ enters in (7.2) only through operators $\tilde{S}(t)$ and (1$\chi(t))\left(S^{p}(t)-\mathrm{Id}\right)$ for which we have Gaussian decay in $L^{p}(\mathbb{R})$ with respect to ${ }^{13}\left\|\partial_{x} h_{0}\right\|_{L^{1}(\mathbb{R}) \cap H^{1}(\mathbb{R})}$, hence the error incurred by defining $\psi$ by (7.1) instead of the value $\tilde{\psi}(t)=s^{p}(t)\left(h_{0} \bar{u}^{\prime}+d_{0}\right)+$ $\int_{0}^{t} s^{p}(t-s) \mathcal{N}(s) d s$ exactly canceling $s^{p}$ terms is harmless to our analysis. The choice of (7.1) reflects our need to accomodate the incompatibility between the initial value $\left.\psi\right|_{t=0}=h_{0}$ prescribed by the spatially-asymptotic behavior of the initial perturbation and the function $\tilde{\psi}$ encoding timeasymptotic behavior of the perturbed solution; that is, it is a device to avoid having to resolve an initial layer near $t=0 .{ }^{14}$ Whether this initial layer is an artifact of our analysis or reflects some aspect of short-time behavior is unclear; as the estimates show, this is below our level of resolution.

\section{Nonlinear STABILITY}

With these preparations, the proof of stability now goes essentially as in the localized conservative case treated in [JZ2], using the new linear modulation bounds to estimate the new linear term coming from data $h_{0} \bar{u}^{\prime}$ in (7.1) and (7.2). As noted in [JZ1], from differential equation (5.2) together with integral equation (7.2) (7.1), we readily obtain short-time existence and continuity with respect to $t$ of solution $\left(v, \psi_{t}, \psi_{x}\right) \in H^{K}(\mathbb{R})$ by a standard contraction-mapping argument

\footnotetext{
${ }^{13}$ For simplicity we set $l=m=0$ in this discussion.

${ }^{14}$ In the case $h_{0} \equiv 0$, this essentially reduces to the simpler device used in [JZ1] to treat the localized case, of substituting $\chi(t) s^{p}(t)$ for $s^{p}(t)$. However, the latter is clearly too crude to treat the present case.
} 
treating the linear $d f(\bar{u}) v$ term of the lefthand side along with $\mathcal{Q}, \mathcal{R}, \mathcal{S}, \mathcal{T}, \psi \bar{u}^{\prime}$ terms of the righthand side as sources in the heat equation. Associated with this solution define so long as it is finite,

$$
\zeta(t):=\sup _{0 \leq s \leq t}\left\|\left(v, \psi_{t}, \psi_{x}\right)(s)\right\|_{H^{K}(\mathbb{R})}(1+s)^{1 / 4} .
$$

Lemma 8.1. For all $t \geq 0$ for which $\zeta(t)$ is finite and sufficiently small, some $C>0$, and $E_{0}:=\left\|\left(d_{0}, \partial_{x} h_{0}\right)\right\|_{L^{1}(\mathbb{R}) \cap H^{K}(\mathbb{R})}$ sufficiently small,

$$
\zeta(t) \leq C\left(E_{0}+\zeta(t)^{2}\right) .
$$

Proof. ${ }^{15}$ By (5.3)-(5.6) and corresponding bounds on the derivatives together with definition (8.1), and using (6.2) to bound $v_{t}$,

$$
\|\mathcal{N}(t)\|_{L^{1}(\mathbb{R}) \cap H^{1}(\mathbb{R})} \lesssim\left\|\left(v, \psi_{t}, \psi_{x}\right)(t)\right\|_{H^{3}(\mathbb{R})}^{2} \leq C \zeta(t)^{2}(1+t)^{-\frac{1}{2}},
$$

so long as $\zeta(t)$ remains small. Applying the bounds (3.5)(1)-(3.6)(1) and (4.1)-(4.3) of Propositions 3.1 and 4.1 to system (7.1)- (7.2), we obtain for any $2 \leq p<\infty$

$$
\begin{aligned}
\|v(t)\|_{L^{p}(\mathbb{R})} & \leq C(1+t)^{-\frac{1}{2}(1-1 / p)} E_{0}+C \zeta(t)^{2} \int_{0}^{t}(1+t-s)^{-\frac{1}{2}(1-1 / p)-\frac{1}{2}}(1+s)^{-\frac{1}{2}} d s \\
& \leq C_{p}\left(E_{0}+\zeta(t)^{2}\right)(1+t)^{-\frac{1}{2}(1-1 / p)}
\end{aligned}
$$

and

$$
\begin{aligned}
\left\|\left(\psi_{t}, \psi_{x}\right)(t)\right\|_{W^{K+1, p}(\mathbb{R})} & \leq C(1+t)^{-\frac{1}{2}} E_{0}+C \zeta(t)^{2} \int_{0}^{t}(1+t-s)^{-\frac{1}{2}(1-1 / p)-1 / 2}(1+s)^{-\frac{1}{2}} d s \\
& \leq C_{p}\left(E_{0}+\zeta(t)^{2}\right)(1+t)^{-\frac{1}{2}(1-1 / p)} .
\end{aligned}
$$

Estimate (8.5) yields in particular that $\left\|\left(\psi_{t}, \psi_{x}\right)(t)\right\|_{H^{K+1}(\mathbb{R})}$ is small, verifying the hypotheses of Proposition 6.1. From (6.1) and (8.4)-(8.5), we thus obtain

$$
\|v(t)\|_{H^{K}(\mathbb{R})} \leq C\left(E_{0}+\zeta(t)^{2}\right)(1+t)^{-\frac{1}{4}} .
$$

Combining this with (8.5), $p=2$, rearranging, and recalling definition (8.1), we obtain the result.

Proof of Theorem 1.1. By short-time $H^{K}$ existence theory, $\left\|\left(v, \psi_{t}, \psi_{x}\right)(t)\right\|_{H^{K}(\mathbb{R})}$ is continuous so long as it remains small, hence $\zeta$ remains continuous so long as it remains small. By (8.1), therefore, it follows by continuous induction that $\zeta(t) \leq 2 C E_{0}$ for $t \geq 0$, if $E_{0}<1 / 4 C$, yielding by (8.1) the result (1.7) for $p=2$. Applying (8.4)-(8.5), we obtain (1.7) for $2 \leq p \leq p_{*}$ for any $p_{*}<\infty$, with uniform constant $C$. Taking $p_{*}>4$ and estimating

$$
\|(\mathcal{Q}, \mathcal{R}, \mathcal{S}, \mathcal{T})(t)\|_{L^{2}(\mathbb{R})} \lesssim\left\|\left(v, \psi_{t}, \psi_{x}, \psi_{x x}\right)(t)\right\|_{L^{4}(\mathbb{R})}^{2} \leq C E_{0}(1+t)^{-\frac{3}{4}}
$$

in place of the weaker (8.3), then applying (3.5)(ii) in place of (3.5)(i), we obtain ${ }^{16}$

$$
\begin{aligned}
\left\|\left(\psi_{t}, \psi_{x}\right)(t)\right\|_{W^{K+1, p}(\mathbb{R})} & \leq C(1+t)^{-\frac{1}{2}} E_{0}+C \zeta(t)^{2} \int_{0}^{t}(1+t-s)^{-\frac{1}{2}(1 / 2-1 / p)-1 / 2}(1+s)^{-\frac{3}{4}} d s \\
& \leq C\left(E_{0}+\zeta(t)^{2}\right)(1+t)^{-\frac{1}{2}(1-1 / p)},
\end{aligned}
$$

\footnotetext{
${ }^{15}$ Compare to the argument of Lemma 4.2, [JZ2], regarding localized perturbations in the conservative case.

${ }^{16}$ We bound the $\partial_{t} \mathcal{S}$ contribution according to

$$
\int_{0}^{t} s^{p}(t-s) \partial_{t} \mathcal{S}(s) d s=-\int_{0}^{t} \partial_{t}\left[s^{p}\right](t-s) \mathcal{S}(s) d s+s^{p}(0) \mathcal{S}(t)-s^{p}(t) \mathcal{S}(0)
$$
}


for $2 \leq p \leq \infty$. Likewise, using (8.6) together with bound

$$
\|(\mathcal{Q}, \mathcal{T})(t)\|_{H^{1}(\mathbb{R})}+\|\mathcal{R}(t)\|_{H^{2}(\mathbb{R})}+\|\mathcal{S}(t)\|_{H^{3}(\mathbb{R})} \lesssim \zeta(t)^{2}(1+t)^{-\frac{1}{2}}
$$

obtained in the course of proving (8.3), we may use (3.6)(ii) rather than (3.6)(i) to get

$$
\begin{aligned}
\|v(t)\|_{L^{p}(\mathbb{R})} & \leq C(1+t)^{-\frac{1}{2}(1-1 / p)} E_{0}+C \zeta(t)^{2} \int_{0}^{t} e^{-\eta(t-s)}(1+s)^{-\frac{1}{2}} d s \\
& +C \zeta(t)^{2} \int_{0}^{t}(1+t-s)^{-\frac{1}{2}(1 / 2-1 / p)-\frac{1}{2}}(1+s)^{-\frac{3}{4}} d s \\
& \leq C\left(E_{0}+\zeta(t)^{2}\right)(1+t)^{-\frac{1}{2}(1-1 / p)}
\end{aligned}
$$

and achieve the proof of (1.7) for $2 \leq p \leq \infty$.

Estimate (1.8) then follows through (7.1) using (3.5)(i), by

$$
\|\psi(t)\|_{L^{\infty}(\mathbb{R})} \leq C E_{0}+C \zeta(t)^{2} \int_{0}^{t}(1+t-s)^{-\frac{1}{2}}(1+s)^{-\frac{1}{2}} d s \leq C\left(E_{0}+\zeta(t)^{2}\right),
$$

yielding nonlinear stability by the fact that

$$
\tilde{u}(x-\psi(x, t), t)-\bar{u}(x-\psi(x, t))=v(x, t)+\bar{u}(x)-\bar{u}(x-\psi(x, t)),
$$

so that $|\tilde{u}(t)-\bar{u}|$ is controlled by the sum of $|v(t)|$ and $|\bar{u}-\bar{u}(\cdot-\psi(\cdot, t))| \lesssim|\psi(t)| \sup \left|\bar{u}^{\prime}\right|$.

Acknowledgement. Thanks to Bjorn Sandstede for pointing out the results of [SSSU].

\section{REFERENCES}

[BJNRZ1] B. Barker, M. Johnson, P. Noble, M. Rodrigues, and K. Zumbrun, Witham averaged equations and modulational stability of periodic solutions of hyperbolic-parabolic balance laws, to appear, Proceedings and seminars, Centre de Mathématiques de l'École polytechnique; Conference proceedings, "Journées equations aux derivées partielles", 2010, Port d'Albret, France.

[BJNRZ2] B. Barker, M. Johnson, P. Noble, M. Rodrigues, and K. Zumbrun, Stability of periodic KuramotoSivashinsky waves, to appear, Appl. Math. Letters.

[BJNRZ3] B. Barker, M. Johnson, P. Noble, M. Rodrigues, and K. Zumbrun, Nonlinear modulational stability of periodic traveling-wave solutions of the generalized Kuramoto-Sivashinsky equation, in preparation.

[DSSS] A. Doelman, B. Sandstede, A. Scheel, and G. Schneider, The dynamics of modulated wavetrains, Mem. Amer. Math. Soc. 199 (2009), no. 934, viii+105 pp. ISBN: 978-0-8218-4293-5.

[G] R. Gardner, On the structure of the spectra of periodic traveling waves, J. Math. Pures Appl. 72 (1993), 415-439.

[JNRZ1] M. Johnson, P. Noble, L.M. Rodrigues, and K. Zumbrun, Nonlocalized modulation of periodic reaction diffusion waves: The Whitham equation, Preprint (2011).

[JNRZ2] M. Johnson, P. Noble, L.M. Rodrigues, and K. Zumbrun, Modulation and large time asymptotic profiles for viscous roll waves, in preparation.

[JNRZ3] M. Johnson, P. Noble, L.M. Rodrigues, and K. Zumbrun, Behavior of periodic solutions of viscous conservation laws under localized and nonlocalized perturbations, in preparation.

[JZ1] M. Johnson and K. Zumbrun, Nonlinear stability of spatially-periodic traveling-wave solutions of systems of reaction diffusion equations, to appear, Annales de l'Institut Henri Poincaré - Analyse non linéaire.

[JZ2] M. Johnson and K. Zumbrun, Nonlinear stability of periodic traveling waves of viscous conservation laws in the generic case, Journal of Differential Equations, 249 (2010), no. 5, 1213-1240.

[JZN] M. Johnson, K. Zumbrun, and P. Noble, Nonlinear stability of viscous roll waves, SIAM J. Math. Anal. 43 (2011), no. 2, 557-611.

[K] T. Kato, Perturbation theory for linear operators, Springer-Verlag, Berlin Heidelberg (1985).

[NR1] P. Noble, and M. Rodrigues, Whitham's modulation equations for shallow flows, Preprint (2010).

[NR2] P. Noble, and M. Rodrigues, Modulational stability of periodic waves of the generalized KuramotoSivashinsky equation, Preprint (2010).

[OZ1] M. Oh, and K. Zumbrun, Stability and asymptotic behavior of traveling-wave solutions of viscous conservation laws in several dimensions, Arch. Ration. Mech. Anal. 196 (2010), no. 1, 1-20. Erratum: Arch. Ration. Mech. Anal., 196 (2010), no. 1, 21-23. 
[OZ2] M. Oh, and K. Zumbrun, Low-frequency stability analysis of periodic traveling-wave solutions of viscous conservation laws in several dimensions, Z. Anal. Anwend. 25 (2006), no. 1, 1-21.

[Pr] J. Prüss, On the spectrum of $C^{0}$-semigroups. Trans. Amer. Math. Soc. 284 (1984), no. 2, $847-857$.

[SSSU] B. Sandstede, A. Scheel, G. Schneider, and H. Uecker, Diffusive mixing of periodic wave trains in reactiondiffusion systems with different phases at infinity, Preprint (2011).

[S1] G. Schneider, Nonlinear diffusive stability of spatially periodic solutions-abstract theorem and higher space dimensions, Proceedings of the International Conference on Asymptotics in Nonlinear Diffusive Systems (Sendai, 1997), 159-167, Tohoku Math. Publ., 8, Tohoku Univ., Sendai, 1998.

[S2] G. Schneider, Diffusive stability of spatial periodic solutions of the Swift-Hohenberg equation, (English. English summary) Comm. Math. Phys. 178 (1996), no. 3, 679-702.

[Se] D. Serre, Spectral stability of periodic solutions of viscous conservation laws: Large wavelength analysis, Comm. Partial Differential Equations 30 (2005), no. 1-3, 259-282.

[U] H. Uecker, Diffusive stability of rolls in the two-dimensional real and complex Swift-Hohenberg equation, Comm. Partial Differential Equations 24 (1999), no. 11-12, 2109-2146.

INDIANA UNIVERSITY, BLOOMINGTON, IN 47405

E-mail address: matjohn@indiana.edu

Université Lyon I, Villeurbanne, France

E-mail address: noble@math.univ-lyon1.fr

Université LyOn 1, Villeurbanne, France

E-mail address: rodrigues@math.univ-lyon1.fr

INDIANA UNIVERSITy, BLOOMINGTON, IN 47405

E-mail address: kzumbrun@indiana.edu 